Old Dominion University

ODU Digital Commons

Mechanical \& Aerospace Engineering Faculty

Publications

Mechanical \& Aerospace Engineering

2020

\title{
Toward Adjoint-Based Aeroacoustic Optimization for Propeller and Rotorcraft Applications
}

\author{
Ramiz Ö. Içke \\ Old Dominion University \\ Oktay Baysal \\ Old Dominion University, obaysal@odu.edu \\ Andy Moy \\ Old Dominion University, amoy003@odu.edu \\ Leonard V. Lopes \\ Beckett Zhou
}

See next page for additional authors

Follow this and additional works at: https://digitalcommons.odu.edu/mae_fac_pubs

Part of the Aerodynamics and Fluid Mechanics Commons, and the Systems Engineering and Multidisciplinary Design Optimization Commons

\section{Original Publication Citation}

Içke, R. Ö., Baysal, O., Moy, A., Lopes, L. V., Zhou, B., \& Diskin, B. D. (2020). Toward adjoint-based aeroacoustic optimization for propeller and rotorcraft applications. AIAA Aviation 2020 Forum, Virtual, June 15-19, 2020. https://doi.org/10.2514/6.2020-3140

This Conference Paper is brought to you for free and open access by the Mechanical \& Aerospace Engineering at ODU Digital Commons. It has been accepted for inclusion in Mechanical \& Aerospace Engineering Faculty Publications by an authorized administrator of ODU Digital Commons. For more information, please contact digitalcommons@odu.edu. 


\section{Authors}

Ramiz Ö. Içke, Oktay Baysal, Andy Moy, Leonard V. Lopes, Beckett Zhou, and Boris Diskin 


\title{
Toward Adjoint-Based Aeroacoustic Optimization for Propeller and Rotorcraft Applications
}

\author{
R. Omur Icke ${ }^{1}$, Oktay Baysal ${ }^{2}$ and Andy Moy ${ }^{3}$ \\ Old Dominion University, Norfolk, VA, 23529, USA \\ Leonard Lopes ${ }^{4}$ \\ NASA Langley Research Center, Hampton, VA, 23666, USA \\ Beckett Zhou ${ }^{5}$ \\ Chair for Scientific Computing, TU Kaiserslautern, 67663, Germany \\ Boris Diskin 6 \\ National Institute of Aerospace, Hampton, VA, 23666, USA
}

\begin{abstract}
The goal of the present project is to build a multidisciplinary, rapid, robust, and accurate computational tool to optimize wing-mounted propeller designs. The full Farassat's formulation F1A for aeroacoustic analysis is implemented in the open-source software SU2. This extension enables the prediction of far-field noise generated by moving sources. The formulation is verified, for a stationary and rotating sphere in a wind tunnel and for a tiltrotor in forward flight, by comparing the acoustic predictions of SU2 with the predictions computed by NASA's aeroacoustics code ANOPP2. The algorithmic differentiation capability of SU2 provides discretely consistent, adjoint-based sensitivity analysis for this formulation. The adjoint-based sensitivities are verified through comparison with complex-step sensitivities.
\end{abstract}

\section{Nomenclature}

$c \quad=$ speed of sound

$C_{T} \quad=$ thrust coefficient

$H(f)=$ Heaviside function

$J \quad=$ acoustic objective function

$\dot{M}_{i} \quad=$ time derivative of local Mach number

$M \quad=$ Mach number

$n_{i}, n_{j} \quad=$ unit outward normal

$p=$ pressure

$p_{\infty} \quad=$ freestream pressure

$p^{\prime} \quad=$ pressure fluctuation

\footnotetext{
${ }^{1}$ Corresponding Author. PhD Candidate, Mechanical \& Aerospace Engineering, Member AIAA, ricke001@odu.edu.

${ }^{2}$ Professor \& Eminent Scholar, Mechanical \& Aerospace Engineering, Associate Fellow, AIAA, obaysal@ odu.edu.

${ }^{3} \mathrm{PhD}$ Candidate, Mechanical \& Aerospace Engineering, Member AIAA, amoy003@ odu.edu.

${ }^{4}$ Research Aerospace Engineer, Aeroacoustics Branch, MS 461, Leonard.V.Lopes@ nasa.gov.

${ }^{5}$ Research Scientist, Member AIAA, yuxiang.zhou@scicomp.uni-kl.de.

${ }^{6}$ Research Fellow, Associate Fellow, AIAA, Boris.Diskin@ nianet.org.
} 


$\begin{array}{ll}p_{R M S}^{\prime} & =\text { root mean square of pressure fluctuation } \\ r & =\text { radiation vector } \\ \hat{r}_{i} & =\text { unit radiation vector } \\ S & =\text { surface area } \\ T_{i j} & =\text { Lighthill stress sensor } \\ v_{i} & =\text { local velocity } \\ v_{n} & =\text { surface velocity } \\ \delta(f) & =\text { Dirac delta function } \\ \delta_{i j} & =\text { Kronecker delta } \\ \square^{2} & =\text { D'Alembert operator } \\ \eta & =\text { propulsion efficiency } \\ \rho_{0} & =\text { freestream density } \\ \sigma & =\text { solidity }\end{array}$

\section{Introduction}

Predicting and reducing the noise emitted by air vehicles is critically important to improve public acceptance of such vehicles for operations in densely populated areas. An accurate prediction of noise is a challenging task requiring a detailed representation of unsteady turbulent flow fields, long-distance propagation of the acoustic disturbances, and evaluation of complex and evolving noise metrics. Optimization of a quiet aircraft design is even more complicated, as it typically involves hundreds of design variables, with few design objectives, such as a noise limitation and adherence to an aerodynamic performance metric. The adjoint method for design enables gradient-based optimization with many design variables and with only a few design objectives. The computational cost of evaluating the adjoint-based sensitivities is independent of the number of design variables [1-3]. Significant progress has been achieved in adjoint-based aerodynamic shape optimization in both steady and unsteady settings [3-11].

Herein, a computational framework is developed and presented to analyze a complex flowfield and acoustic wave propagation generated by propellers and rotary wing aircraft. The framework is based on the SU2-suite, which is an open-source collection of tools for the analysis of partial differential equations (PDEs) and PDE-constrained optimization problems on unstructured meshes with state-of-the-art numerical methods $[8,9,12]$.

The SU2 code has recently been expanded to include a computational aeroacoustic (CAA) model using a Ffowcs Williams and Hawkings (FWH) formulation. This model offers an efficient approach for calculating acoustic pressure at arbitrary observer locations by performing boundary integrals once the required field data are provided. In this manner, the radiated noise can be calculated given the near-field flow data supplied by a CFD solution. Demonstrations of SU2-based aeroacoustic analyses, for stationary sources only, have previously been reported [1315].

The focus of this paper is developing and demonstrating the noise prediction and adjoint-based sensitivity analysis capability to optimize propeller and rotorcraft designs. In the present study, Farassat's Formulation-1A (F1A) [16] is adopted for the acoustic wave propagation. As technology demonstrators, the paper reports first on predictions of noise generated by a rotating, impermeable sphere, then by a tiltrotor, both in forward flight. The accuracy of SU2 noise predictions is verified by comparing with static pressure fluctuations at the CFD grid nodes and with the predictions obtained by NASA's next generation Aircraft Noise Prediction Program (ANOPP2) [17, 18]. ANOPP2 is a widely used aircraft noise prediction toolkit that includes a framework, noise-prediction methods, and peripheral software to predict and understand aircraft noise. To enable verification, an interface between SU2 and ANOPP2 has been developed that allows for the transfer of CFD data into ANOPP2 in order to initiate the analysis of noise propagation.

The contents of the paper are presented in the following order. In Section III, the F1A formulation is summarized. The CFD and CAA analyses are presented and compared for verification in Section IV. The adjointbased sensitivities computed by an algorithmic differentiation (AD) method of SU2 are compared with complex-step sensitivities in Section V. The conclusions drawn from the present study and the future work overview are provided in Section VI. 


\section{Methodology}

\section{A. CFD Analysis for Noise Generation and its Sensitivities}

The software SU2 solves the Reynolds-averaged Navier-Stokes (RANS) equations to analyze compressible, turbulent flows commonly found in aerospace engineering problems. The governing equations are spatially discretized using a finite volume method. The turbulence can be either modeled by the Spalart-Allmaras or the Menter Shear Stress Transport model [12]. In the present study, the Jameson-Schimdt-Turkel scheme, with the Green-Gauss theorem for the gradients, is employed for the flux discretizations [19]. The propeller-generated noise requires resolving the unsteadiness. Therefore, the time marching of the semidiscretized Unsteady Reynoldsaveraged Navier-Stokes (URANS) equations is performed by a dual time-stepping method.

A powerful feature of SU2 is the availability of AD rendered by the tool CoDiPack [20]. By successive applications of the chain-rule differentiation through the SU2 code, both the analysis output and its derivative with respect to prescribed design variables are computed simultaneously. A remarkable feature of AD, owing to its construction, is that it does not incur any truncation errors, rendering derivatives that are at machine accuracy.

\section{B. CAA Solver for Noise Propagation}

The FWH equation (Eq. 1) is an alternative form of conservation laws [21]. It includes monopole, dipole, and quadrupole terms with each corresponding to a different source of aerodynamic noise:

$$
\square^{2} p^{\prime}=\frac{\partial}{\partial t}\left[\rho_{0} v_{n} \delta(f)\right]-\frac{\partial}{\partial x_{i}}\left[p n_{i} \delta(f)\right]+\frac{\partial^{2}}{\partial x_{i} \partial x_{j}}\left[H(f) T_{i j}\right],
$$

where $p^{\prime}$ is acoustic pressure, $\square^{2}$ is the D'Alembertian operator and $f(x, t)=0$ represents a moving control surface where $n_{i}$ is the unit outward normal. $H(f)$ and $\delta(f)$ are the Heaviside and Dirac delta functions, respectively. Last, $\rho_{0}, v_{n}, p$ and $T_{i j}$ are defined as freestream density, surface velocity dotted with the surface normal vector, the static pressure and Lighthill stress tensor, respectively. Farassat derived a family of more practical formulations [16] of the FWH equation. In the F1A formulation, the quadrupole term is neglected, and pressure fluctuation is equal to the sum of thickness noise and loading noise [22],

$$
p^{\prime}(\boldsymbol{x}, t)=p_{T}^{\prime}(\boldsymbol{x}, t)+p_{L}^{\prime}(\boldsymbol{x}, t),
$$

where $p_{T}^{\prime}$ and $p_{L}^{\prime}$ are the contributions of the thickness noise and the loading noise, respectively. These terms consist of integral equations as shown in Eqs. (3) and (4):

$$
\begin{gathered}
p_{T}^{\prime}(\mathbf{x}, \mathrm{t})=\frac{1}{4 \pi} \int_{S}\left[\frac{\rho_{0}\left(\dot{U}_{i} n_{i}+U_{i} \dot{n}_{i}\right)}{r\left(1-M_{r}\right)^{2}}\right]_{r e t} d S+\frac{1}{4 \pi} \int_{S}\left[\frac{\rho_{0} U_{i} n_{i} K}{r^{2}\left(1-M_{r}\right)^{3}}\right]_{r e t} d S, \\
p_{L}^{\prime}(\mathbf{x}, \mathrm{t})=\frac{1}{4 \pi} \frac{1}{c} \int_{S}\left[\frac{\dot{F}_{i} \hat{r}_{i}}{r\left(1-M_{r}\right)^{2}}\right]_{r e t} d S+\frac{1}{4 \pi} \int_{S}\left[\frac{F_{i} \hat{r}_{i}-F_{i} M_{i}}{r^{2}\left(1-M_{r}\right)^{2}}\right]_{r e t} d S+\frac{1}{4 \pi} \frac{1}{c} \int_{S}\left[\frac{F_{i} \hat{r}_{i} K}{r^{2}\left(1-M_{r}\right)^{3}}\right]_{r e t} d S,
\end{gathered}
$$

where, for an impermeable surface,

$$
\begin{gathered}
U_{i}=v_{i} \\
F_{i}=L_{i j} n_{j} \\
L_{i j}=P_{i j}^{\prime}=\left(p-p_{\infty}\right) \delta_{i j} \\
K=\dot{M}_{i} \hat{r}_{i} r+M_{r} c-M^{2} c \\
M_{r}=\frac{v_{i}}{c} \hat{r}_{i} .
\end{gathered}
$$


Here, $r$ is the radiation vector or distance between observer and source points. Similarly, $\hat{r}_{i}$ represents the unit radiation vector. $\dot{M}_{i}$ is the time derivative of local Mach number, $M$, depending on speed of sound, $c$, and local velocity, $v_{i}$. In addition, $d S, \delta_{i j}$ and $p_{\infty}$ are the local panel area, the Kronecker delta and the freestream pressure, respectively.

The first FWH implementation in SU2 by Zhou et al. [15], constitutes the initial framework of the developed code. In their study, the far-field acoustic propagation is obtained by utilizing the permeable surface approach only for fixed sources in a "wind tunnel configuration." In the present development, the authors follow the impermeable surface approach again with the time domain implementation as shown in Eqs. (5) - (7). Moreover, the present code is capable of computing moving sources using the full F1A formulation in addition to the previous implementation in Ref. [15].

Based on that formulation, CAA results are obtained and compared with static pressure fluctuations extracted directly from CFD results. The comparison includes, first a stationary, then a moving source, both in a wind tunnel configuration, that is, a stationary observer is placed in a moving medium. This is equivalent to a moving-observer situation with the observer moving at negative freestream velocity, $-\vec{U}_{0}$, in a stationary medium.

\section{Results: Multidisciplinary Analysis by Coupled CFD and CAA}

\section{A. Stationary Source in a Wind Tunnel}

A stationary unit sphere is subjected to the flowfield in a wind tunnel configuration. The rationale behind this choice is the simplicity of the geometry, which allows code debugging in significantly less time, both to generate a mesh and run the case on a computer. The noise is generated by the flow disturbances as they negotiate the curvature of the sphere. Shown in Fig. 1 are the computed pressure field on the sphere surface (Fig. 1a) and its wake (Fig. 1b) for flow at Mach 0.5. Also, to observe the effect of changing the freestream Mach number on the wake flow, the computations are also repeated for Mach 0.1 and Reynolds number $1.14 \times 10^{6}$ [23].

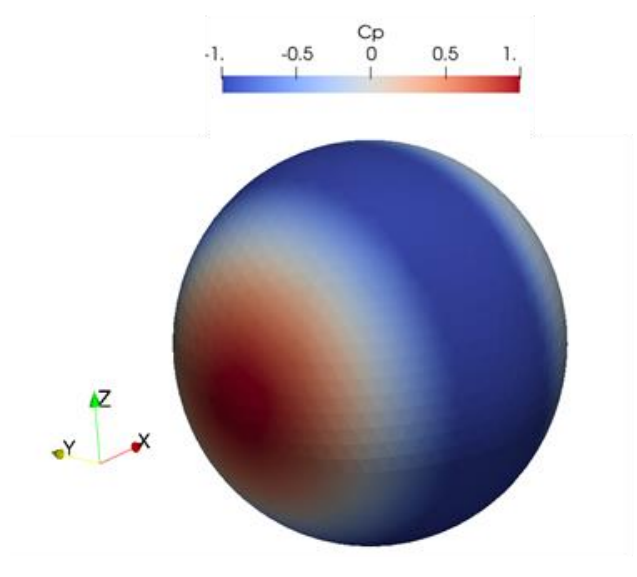

(a)

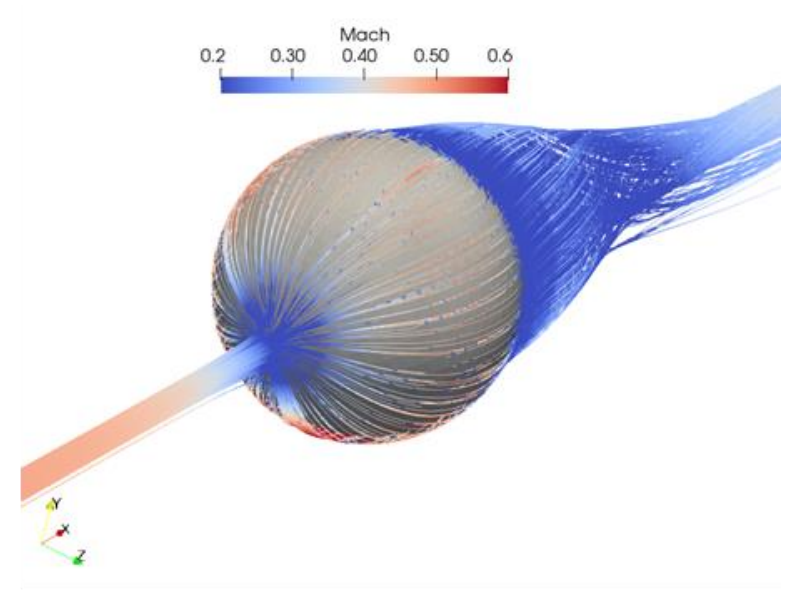

(b)

Fig. 1 (a) Computed pressure coefficient distribution on a unit sphere and (b) its wake.

The pressure fluctuations on the sphere surface, obtained from CFD, are handed over as input to the presently developed CAA routines of the SU2 code. Presented in Figs. 2-5 are the pressure fluctuations propagated to observers at 10 diameters and 14 diameters above the sphere center for Mach numbers 0.5 and 0.1 . The propagation is predicted in two different ways, then compared, first by the present CFD method, then by the F1A formulation (Figs. 2-5). For these low Mach number flows, the CFD results match reasonably those obtained by F1A. It should be noted that the F1A formulation neglects the quadrupole noise terms, which would represent the noise component due to viscous effects and the turbulence. It is expected that, with a denser mesh resolution at the observer location, the comparison should improve, but only until the acoustic signals succumb to the numerical dispersion error of this second-order CFD method. 


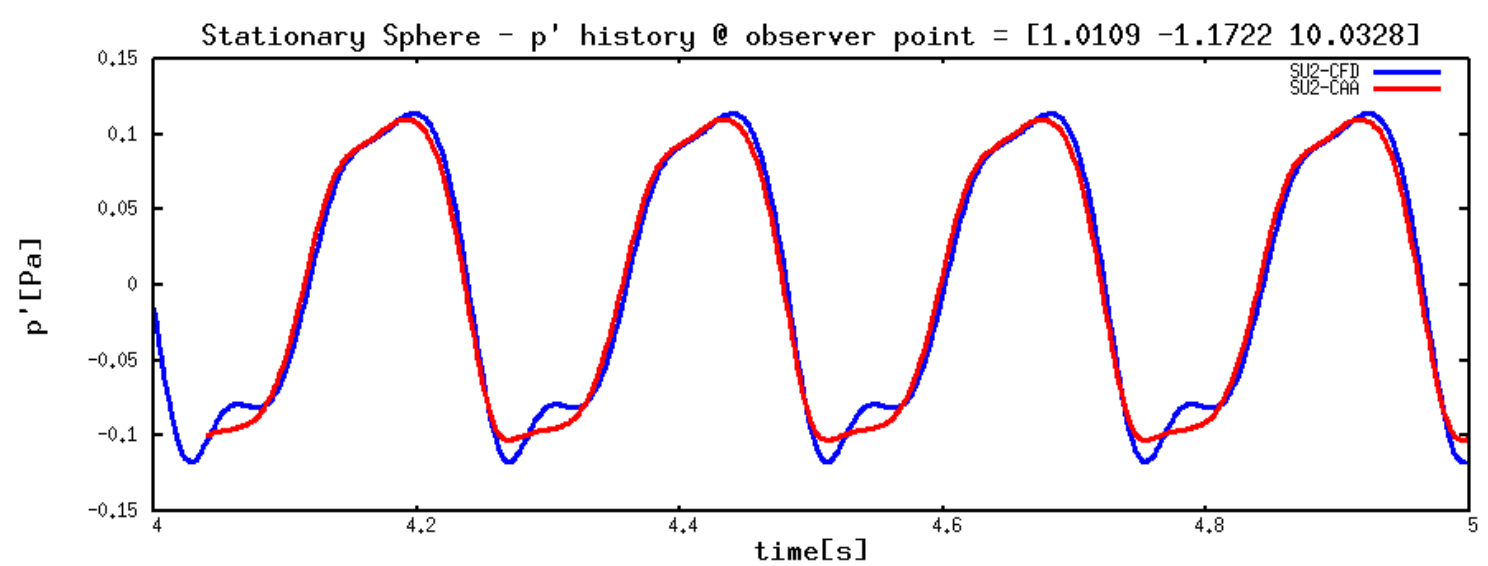

Fig. 2 Comparison of F1A computed pressure propagation with CFD results. $M_{\infty}=0.5$ and the observer at 10 diameters away.

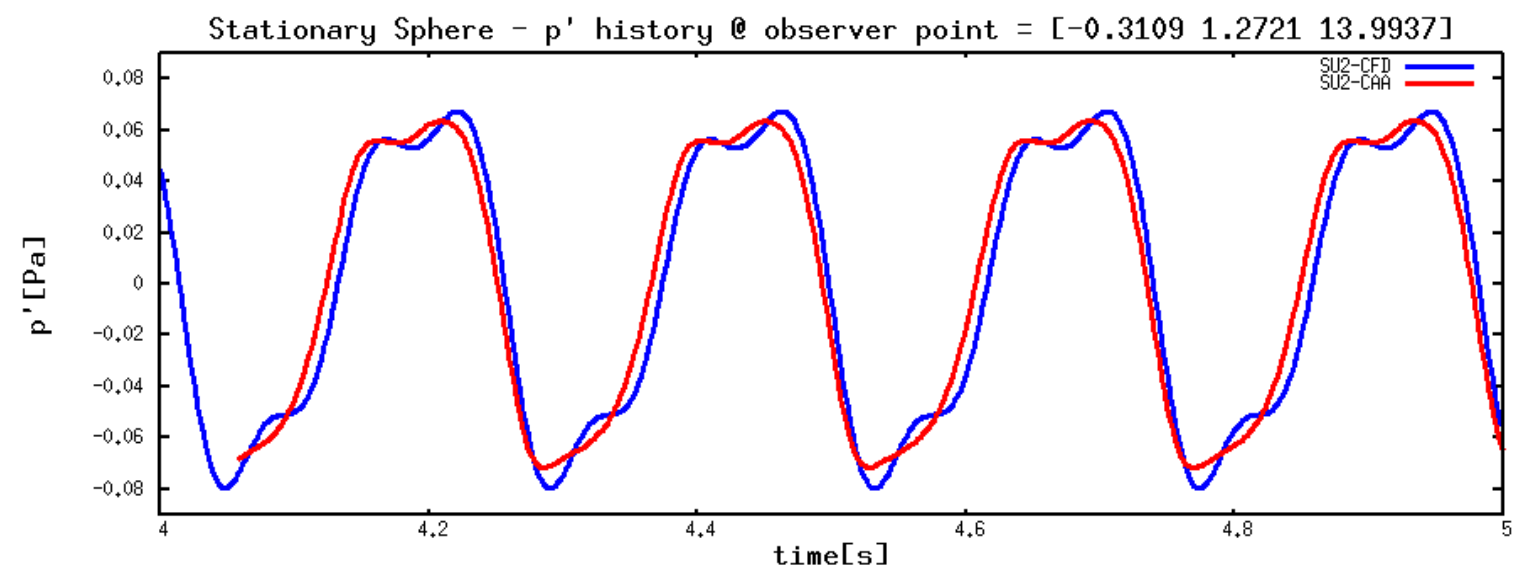

Fig. 3 Comparison of F1A computed pressure propagation with CFD results. $M_{\infty}=0.5$ and the observer at 14 diameters away.

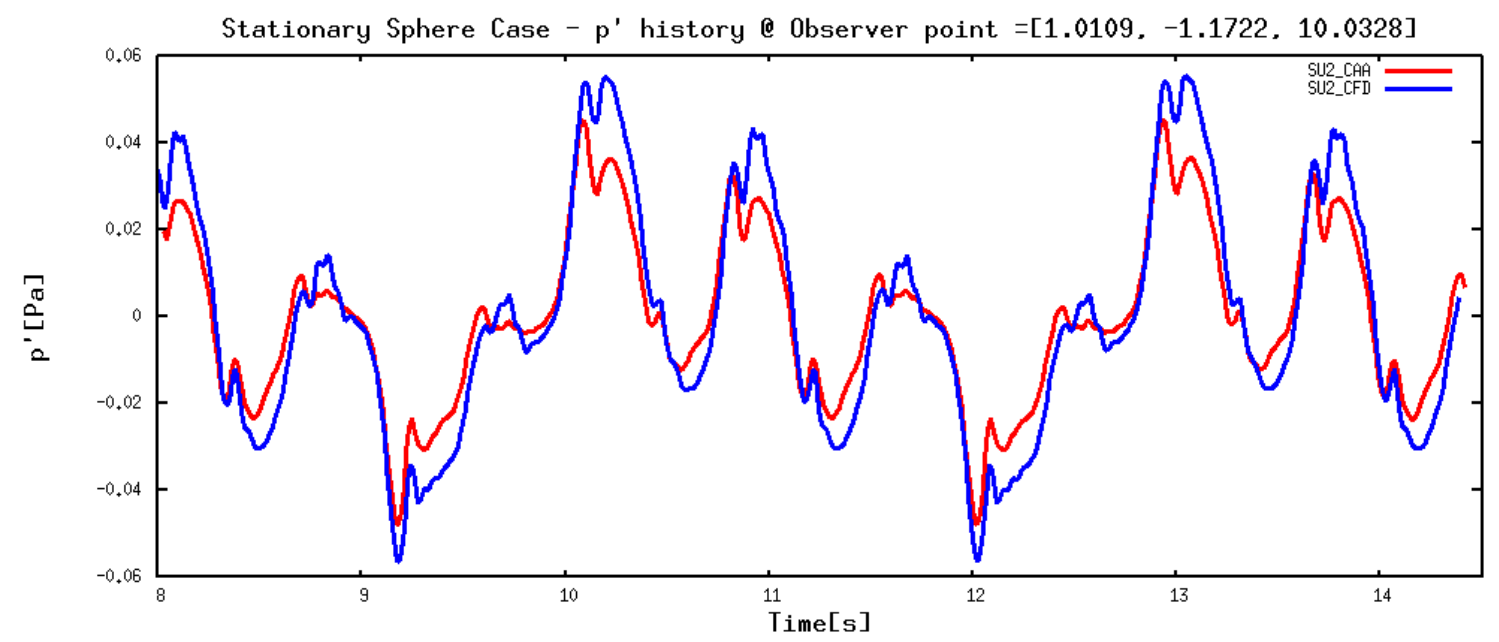

Fig. 4 Comparison of F1A computed pressure propagation with CFD results. $M_{\infty}=0.1$ and the observer at 10 diameters away. 


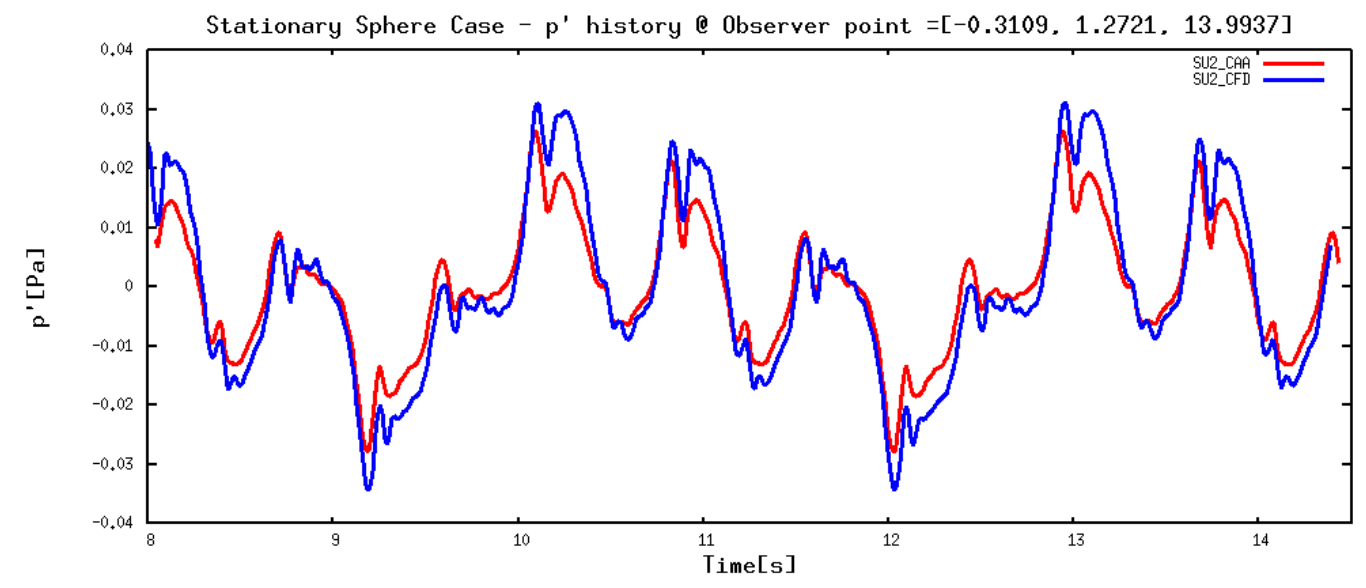

Fig. 5 Comparison of F1A computed pressure propagation with CFD results. $M_{\infty}=0.1$ and the observer at 14 diameters away.

\section{B. Moving Source in a Wind Tunnel}

As mentioned above, the F1A formulation is also valid for moving-source problems. The challenging part proves to be the computations of the surface normal vectors at each time step. In addition to the time derivative of pressure, time derivatives of the normal vectors and velocity vectors, which are not zero in this case, are required.

\section{Rotating Sphere}

The previously described sphere is again considered, but now it is rotating. The rotation is computationally accounted for by rotating the entire CFD mesh as a rigid body. That is, there is no mesh deformation and cells do not move relative to the sphere. For the purpose of developing the code for a rigid body motion, this case appears to be very relevant due to the simplicity of its geometry and its motion.

As in the stationary sphere case, comparisons are made for the fluctuating term computed at the observer location, which is 10 diameters away from the sphere center. Here, the rotating and the freestream Mach numbers are 0.12 and 0.5 , respectively. In the CAA analysis, the observer point is considered fixed, e.g., the coordinates are $[0.0,0.0,10.0]$. Whereas, the virtual pressure probe, recording the computed CFD values, is rotating with the sphere's rigid body motion. Therefore, the probe coordinates are not at the same distance from the source. Consequently, the values gathered from the probe show an oscillatory behavior. This is displayed in Fig. 6, where pressure fluctuations computed from CFD demonstrate jaggedness, while CAA produces a rather smooth distribution.

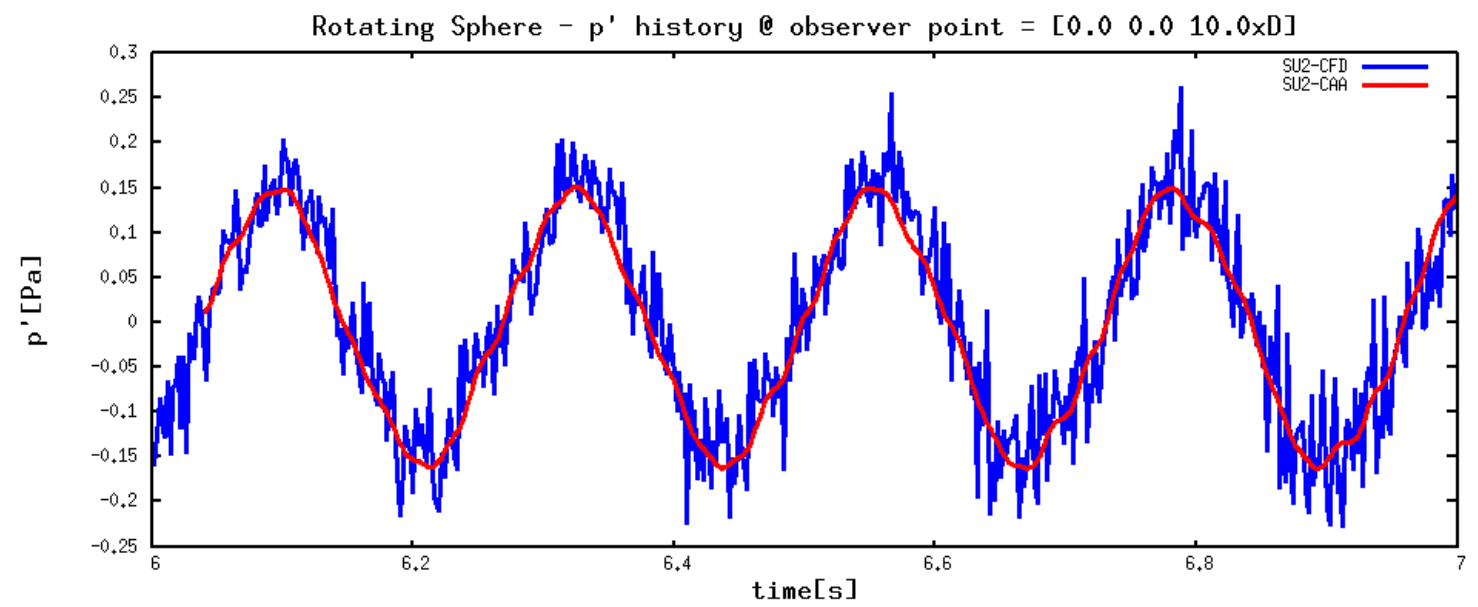

Fig. 6 Comparison of F1A-computed pressure propagation with CFD results for a rotating unit sphere. 


\section{Rotor in Forward Flight}

For further code development, the aerodynamic and the aeroacoustic fields of a rotor are considered. The XV-15 rotor is a relatively simple yet an acceptably good representative geometry, for which data are publicly available (Fig. 7a). Although the geometric details of XV-15 rotor blades are available in the open literature [24-26], the rest of the assembly, that is, the hub and the pylon components, are not available. After making a few assumptions to make up for the missing information, the CAD model and the CFD mesh were generated (Fig 7b).

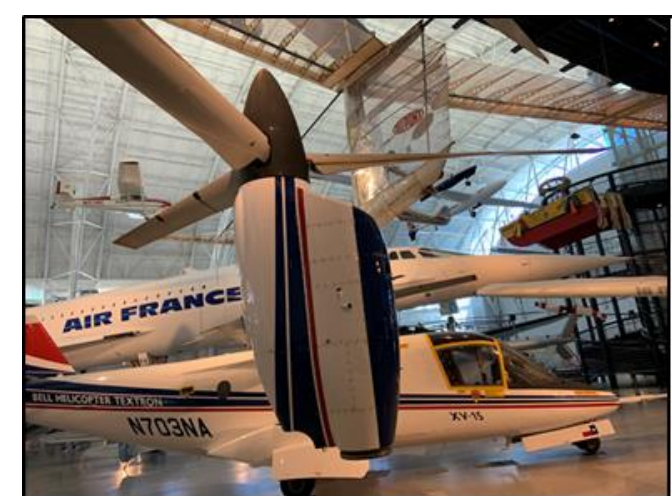

(a)

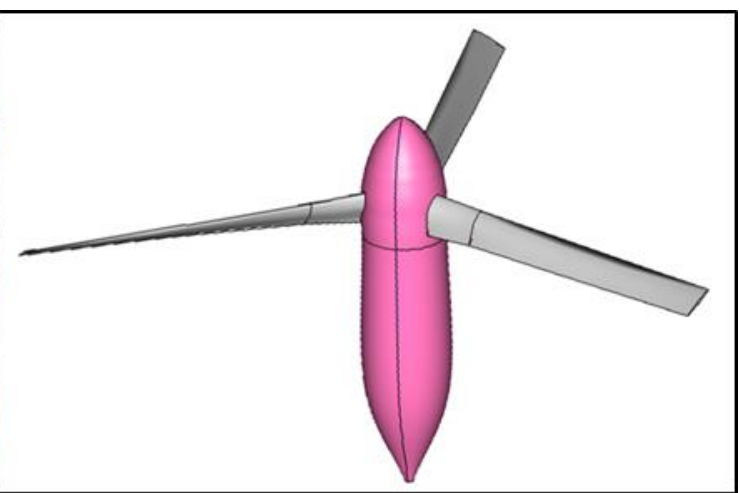

(b)

Fig. 7 (a) Photo of XV-15 rotorcraft; (b) CAD model of XV-15 Rotor.

The propulsive efficiency and the thrust coefficient predicted by the SU2-URANS solver match the experimental data [25] reasonably well (Table 1). As can be observed in Fig. 8, the blade-vortex interaction (BVI) does not exist since this case is in forward flight mode. Hence, the noise generation mechanisms do not include BVI [27]. The CAA computations for the XV-15 in forward flight are observed at the microphone positions shown in Figs. 9a and $9 \mathrm{~b}$. The time history of the acoustic pressure signals at these microphone locations is observed as shown in Figs. 1011.

Table 1: Comparison of CFD and experimental results for $\mathrm{XV}-15$ rotor.

\begin{tabular}{|c|c|c|}
\hline & Experiment $[25]$ & SU2-CFD \\
\hline Efficiency, $\boldsymbol{\eta}$ & 0.9319 & $\mathbf{0 . 8 4 5 6}$ \\
\hline$C_{T} / \boldsymbol{\sigma}$ & $\mathbf{0 . 0 3 7 2}$ & $\mathbf{0 . 0 3 6 7}$ \\
\hline
\end{tabular}




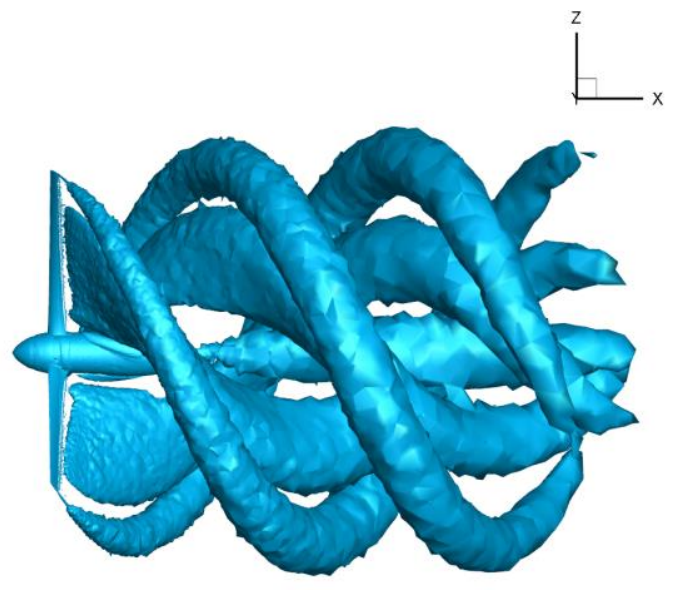

Fig. 8 Instantaneous vorticity isosurfaces (Q-criterion $=2 s^{-2}$ ) in the $\mathrm{XV}-15$ rotor wake.

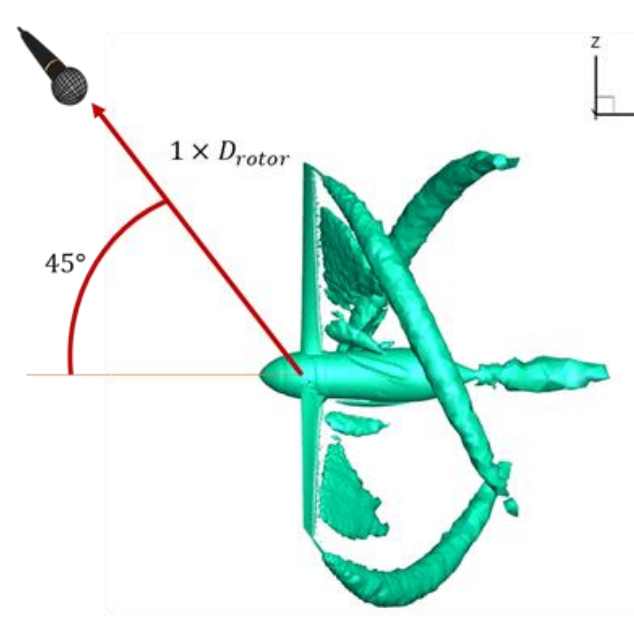

(a)

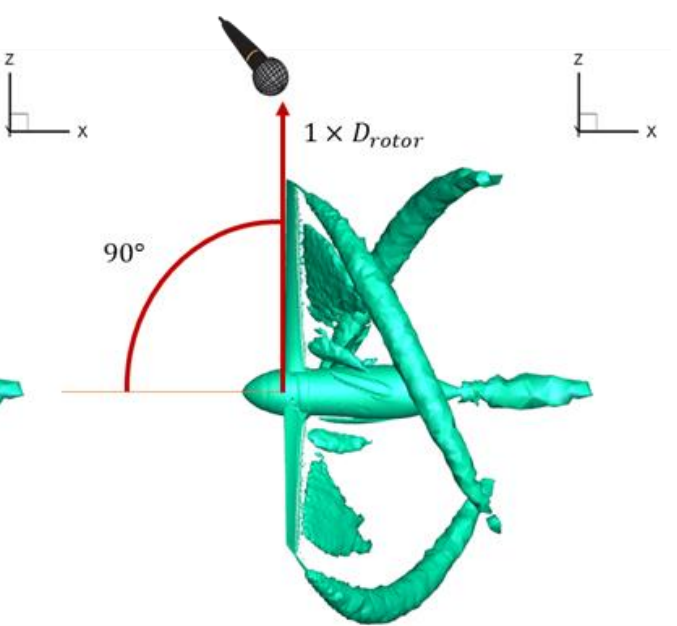

(b)

Fig. 9 Rotor-oriented microphone positions, both one rotor diameter away from the tiltrotor center: (a) Mic 1 located 45 degrees above the rotor plane, and (b) Mic 2 in-plane.

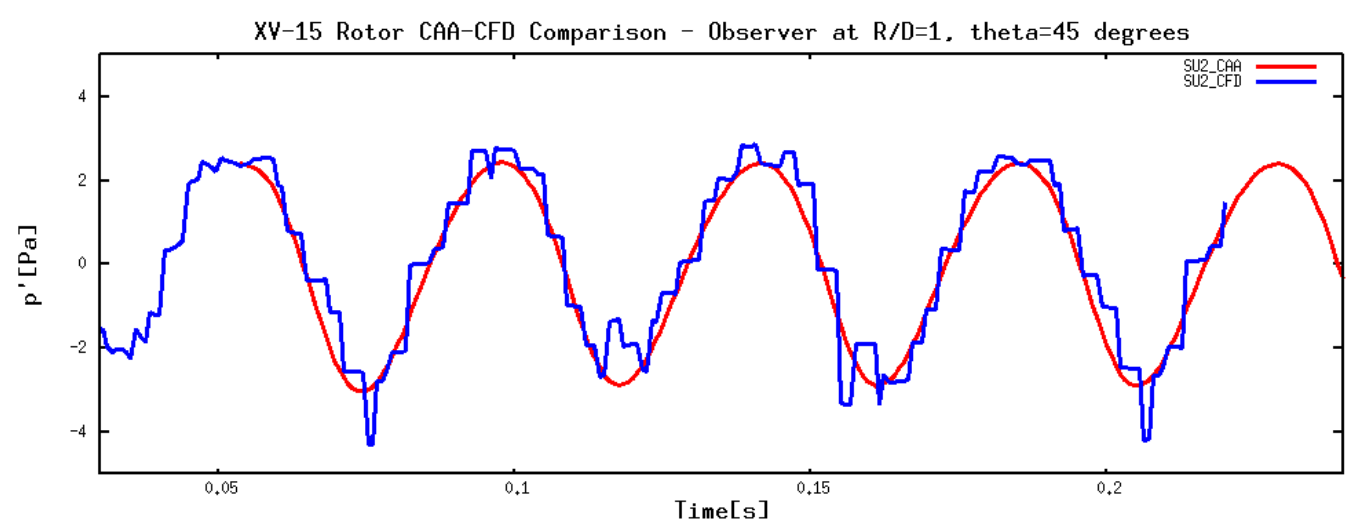

Fig. 10 Comparison of CAA and CFD results for microphone 1 (45 degrees above the rotor plane). 


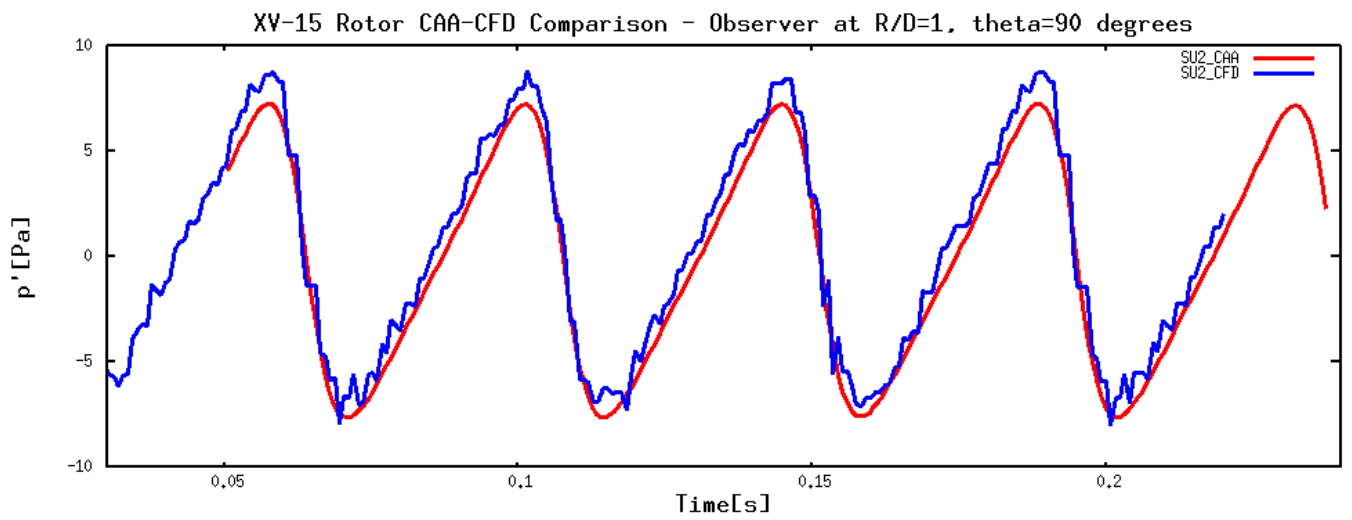

Fig. 11 Comparison of CAA and CFD results for microphone 2 (in-plane position).

Comparison of pressure fluctuations are deemed satisfactory for the tiltrotor case in forward flight. Moreover, the results demonstrate similar characteristics of a propeller described in Ref. [28]. In addition to CAA vs. CFD comparisons, benchmark studies are accomplished using ANOPP2 [17]. As seen in Figs. 12-14, the acoustic pressures obtained from two different software suites (SU2-CAA vs. ANOPP2) demonstrate excellent matches. In this study, SU2-CFD produces flow data in ANOPP2-readable format and transfers to both SU2-CAA and ANOPP2 codes. Note that to make the symbols in Figs. 12-14 easier to view, only every $4^{\text {th }}$ data point is plotted.

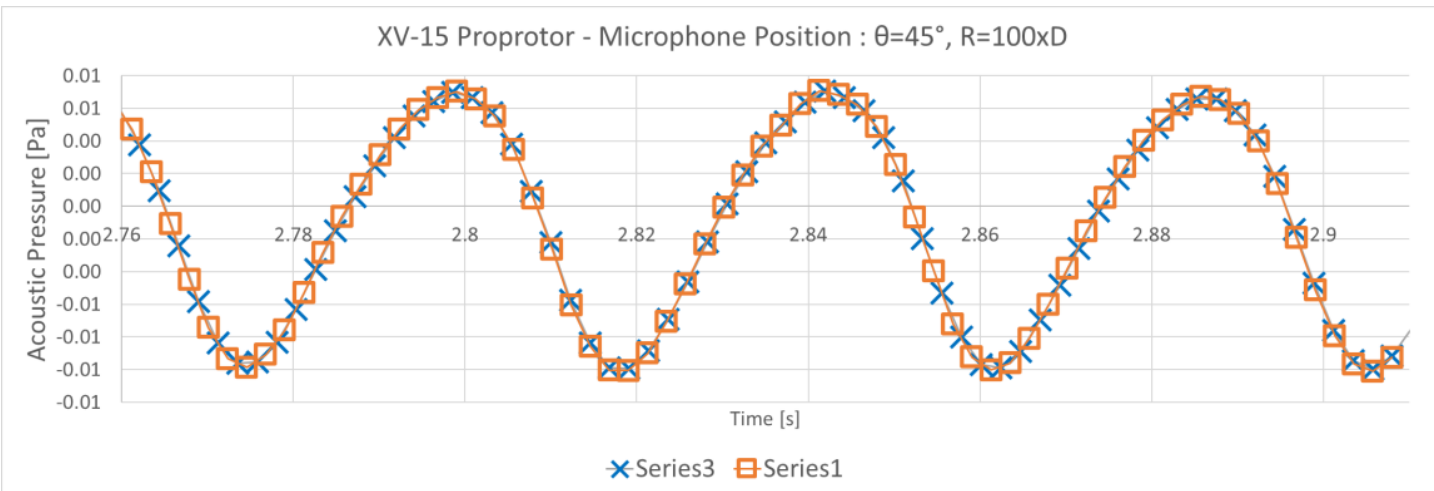

Fig. 12 Acoustic pressure comparison of SU2 and ANOPP2 for the microphone located at 100 diameters away at 45 degrees above the rotor plane.

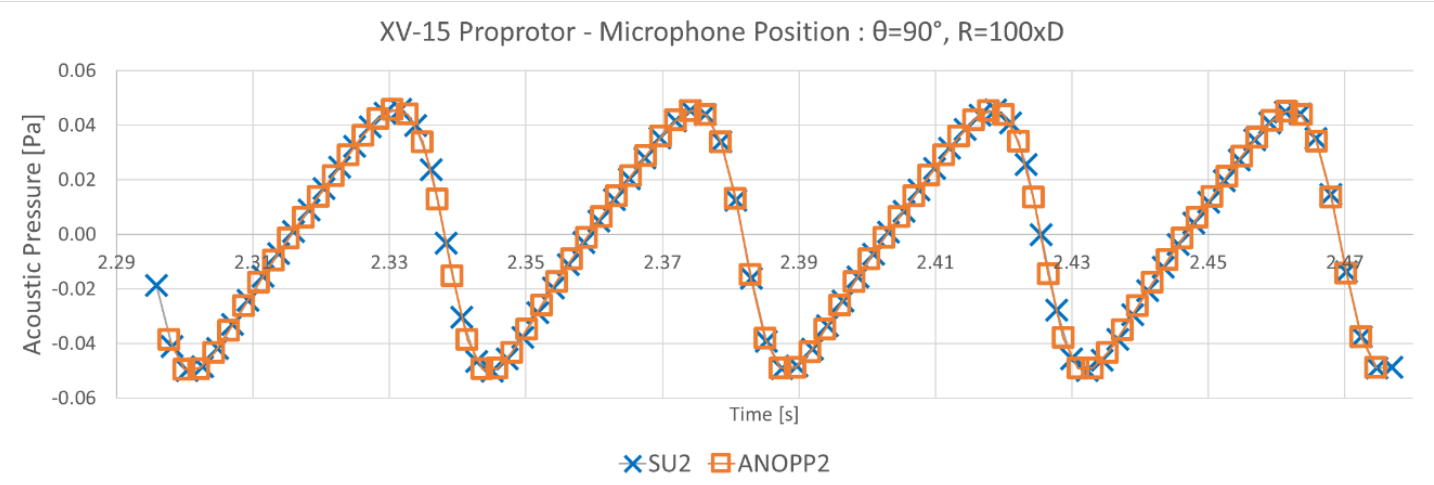

Fig. 13 Acoustic pressure comparison of SU2 and ANOPP2 for the microphone located at 100 diameters away in rotor plane. 


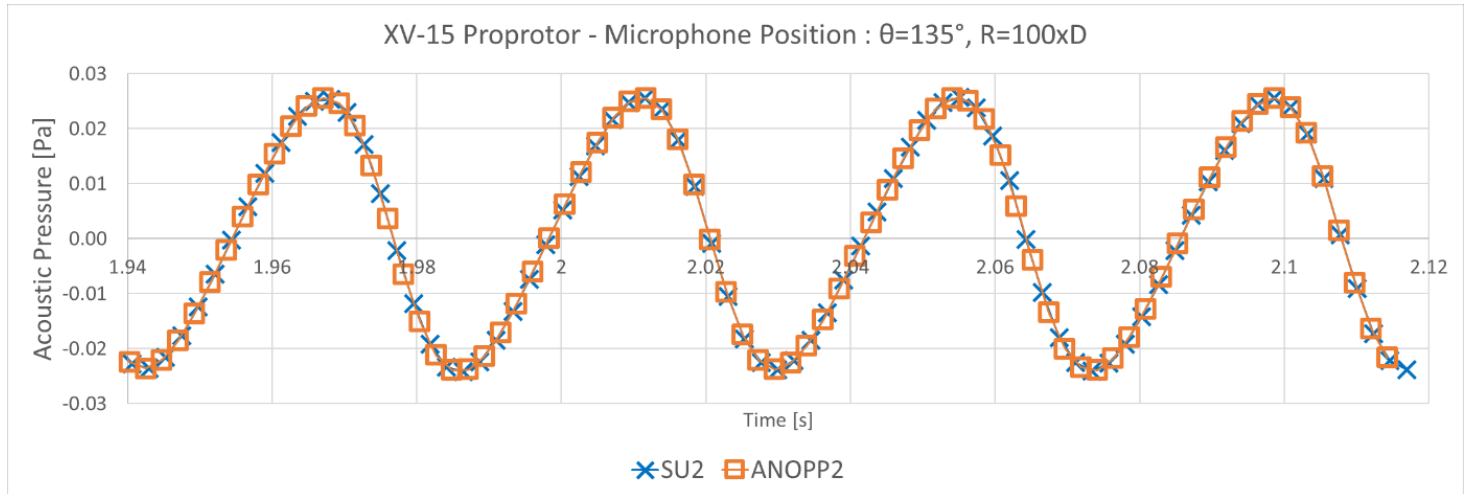

Fig. 14 Acoustic pressure comparison of SU2 and ANOPP2 for the microphone located at 100 diameters away at 45 degrees below the rotor plane.

\section{Results: Adjoint Computation of Sensitivities}

Gradient values with respect to a specific interest, for example, the design parameters, can be efficiently calculated by solving the adjoint equation. The adjoint equations are used in solving numerous engineering problems, such as shape optimization of a wing, placement of flow control devices, and the quantification of numerical uncertainty. In the present study, both the aerodynamic and aeroacoustic gradients are derived by the adjoint approach implemented in the algorithmic differentiation utility CoDiPack [20].

This code includes the adjoint stored for each variable within the panel and the sample loop. Through the process, the recording continues until the adjoint computation is completed. The CoDiPack utility has previously been implemented in SU2 as a built-in function that can be called through the process. Once the adjoint values are stored, the objective function is introduced. For illustrative purposes, let the acoustic objective function, $J$, be defined as the sound pressure level (SPL),

$$
J=S P L=p_{R M S}^{\prime}=\sqrt{\frac{\sum_{n=1}^{N} p^{\prime 2}}{N}} .
$$

One way to verify the accuracy of the gradients obtained by the algorithmic differentiation is to compare them with gradients computed by the complex step-method (Refs. [29, 30]),

$$
F^{\prime}\left(x_{0}\right) \approx \frac{\operatorname{Im}\left(F\left(x_{0}+i h\right)\right)}{h},
$$

where the step value, $h$, is set to $10^{-16}$ for the perturbation. Here the method is demonstrated for two different applications: first, the rotating sphere and, second, the proprotor in forward flight. The results in Table 2 and Table 3 indicate relative errors on the order of $10^{-14}$ or smaller; hence, a successful verification has been achieved. (In the tables below, the first nonmatching digits are identified.)

It should be noted that for an accuracy comparison, it is sufficient to compute the gradients only for one panel with one sample, as shown in these tables. For the sensitivity analysis of the entire domain, with 1,630 panels and 512 samples each, $\mathrm{AD}$ still requires just one calculation (approximate wall clock time is $2.5 \mathrm{~min}$ ). However, $\mathrm{CD}$ has to be restarted for each panel and for each sample, hence it is totally impractical (projected wall clock time would be in thousands of hours). 
Table 2: Rotating Sphere.

\begin{tabular}{|c|c|c|c|}
\hline & \multicolumn{3}{|c|}{ Sensitivities with respect to the grid coordinates } \\
\hline & $\frac{\partial p_{R M S}^{\prime}}{\partial x_{i \text { Panel }=10}^{\text {iSample }=10}}$ & $\frac{\partial p_{R M S}^{\prime}}{\partial y_{i \text { Panpel }=10}^{i \text { Sanple }}}$ & $\frac{\partial p_{R M S}^{\prime}}{\partial z_{\text {iPanplel }=10}^{\text {iSamplo }}}$ \\
\hline Complex & $-1.11711075941204 \mathrm{E}-06$ & $2.59575137516840 \mathrm{E}-06$ & $8.52123831692264 \mathrm{E}-06$ \\
\hline Algorithmic & $-1.11711075941201 \mathrm{E}-06$ & $2.59575137516842 \mathrm{E}-06$ & $8.52123831692268 \mathrm{E}-06$ \\
\hline \multirow[t]{3}{*}{ Relative Error } & $-2.69 \mathrm{E}-14$ & 7.67E-15 & $4.57 \mathrm{E}-15$ \\
\hline & \multicolumn{3}{|c|}{ Sensitivities with respect to the conserved state variables } \\
\hline & $\frac{\partial p_{R M S}^{\prime}}{\partial \rho_{\text {iPanplel }=10}^{i \text { Pamplo }}}$ & $\frac{\partial p_{R M S}^{\prime}}{\partial\left(\rho u_{x}\right)_{i \text { Panple }=10}^{i S a m l e}}$ & $\frac{\partial p_{R M S}^{\prime}}{\partial\left(\rho u_{y}\right)_{i \text { Panel }=10}^{i S a m p l e=10}}$ \\
\hline Complex & $-1.33100974824504 \mathrm{E}-04$ & $-2.54416127718037 \mathrm{E}-06$ & $9.55291842149742 \mathrm{E}-06$ \\
\hline Algorithmic & $-1.33100974824499 \mathrm{E}-04$ & $-2.54416127718029 \mathrm{E}-06$ & $9.55291842149639 \mathrm{E}-06$ \\
\hline \multirow[t]{2}{*}{ Relative Error } & $-3.77 \mathrm{E}-14$ & $-3.15 \mathrm{E}-14$ & $1.08 \mathrm{E}-13$ \\
\hline & $\frac{\partial p_{R M S}^{\prime}}{\partial\left(\rho u_{z}\right)_{i \text { Pample }=10}^{\text {Samplo }}}$ & $\frac{\partial p_{R M S}^{\prime}}{\partial(\rho E)_{\text {iPanele }=10}^{\text {isample }=10}}$ & $\frac{\partial p_{R M S}^{\prime}}{\partial(T K E)_{\text {iPanple }=10}^{i \text { isample }}=10}$ \\
\hline Complex & $2.41024067888670 \mathrm{E}-06$ & $-1.55719679480814 \mathrm{E}-10$ & $1.42096849564304 \mathrm{E}-11$ \\
\hline Algorithmic & $2.41024067888669 \mathrm{E}-06$ & $-1.55719679480818 \mathrm{E}-10$ & $1.42096849564308 \mathrm{E}-11$ \\
\hline Relative Error & $4.22 \mathrm{E}-15$ & $-2.57 \mathrm{E}-14$ & $2.82 \mathrm{E}-14$ \\
\hline
\end{tabular}

Table 3: Proprotor XV-15 in Forward Flight.

\begin{tabular}{|c|c|c|c|}
\hline & \multicolumn{3}{|c|}{ Sensitivities with respect to grid coordinates } \\
\hline & $\frac{\partial p_{R M S}^{\prime}}{\partial x_{i \text { Panpl }=10}^{\text {iSample } 10}}$ & $\frac{\partial p_{R M S}^{\prime}}{\partial y_{\text {iPanple }=10}^{\text {iSample }}}$ & $\frac{\partial p_{R M S}^{\prime}}{\partial z_{\text {iPanel }=10}^{\text {iSample }=10}}$ \\
\hline Complex & $5.2223879126036 \mathrm{E}-08$ & $-2.04617333143159 \mathrm{E}-08$ & $-2.01844927073334 \mathrm{E}-07$ \\
\hline Algorithmic & $5.2223879126041 \mathrm{E}-08$ & $-2.04617333143158 \mathrm{E}-08$ & $-2.01844927073320 \mathrm{E}-07$ \\
\hline \multirow[t]{3}{*}{ Relative Error } & $9.58 \mathrm{E}-14$ & $-4.85 \mathrm{E}-15$ & $-6.92 \mathrm{E}-14$ \\
\hline & \multicolumn{3}{|c|}{ Sensitivities with respect to conserved state variables } \\
\hline & $\frac{\partial p_{R M S}^{\prime}}{\partial \rho_{i \text { Panel }=10}^{i \text { Sample }=10}}$ & $\frac{\partial p_{R M S}^{\prime}}{\partial\left(\rho u_{x}\right)_{\text {iPanel }=10}^{\text {SPample }=10}}$ & $\frac{\partial p_{R M S}^{\prime}}{\partial\left(\rho u_{y}\right)_{i \text { Panel }=10}^{\text {iSample }=10}}$ \\
\hline Complex & $4.06260190032438 \mathrm{E}-07$ & $8.46989723103815 \mathrm{E}-10$ & $2.54290215511708 \mathrm{E}-09$ \\
\hline Algorithmic & $4.06260190032440 \mathrm{E}-07$ & $8.46989723103814 \mathrm{E}-10$ & $2.54290215511706 \mathrm{E}-09$ \\
\hline \multirow[t]{2}{*}{ Relative Error } & $4.95 \mathrm{E}-15$ & $1.22 \mathrm{E}-15$ & 7.97E-15 \\
\hline & $\frac{\partial p_{R M S}^{\prime}}{\partial\left(\rho u_{z}\right)_{\text {iPanel }=10}^{\text {iSample }=10}}$ & $\frac{\partial p_{R M S}^{\prime}}{\partial(\rho E)_{\text {iPanel }=10}^{i \text { Sample }=10}}$ & $\frac{\partial p_{R M S}^{\prime}}{\partial(T K E)_{\text {iPanel }}^{\text {iSample }=10}}$ \\
\hline Complex & $-1.91045438660576 \mathrm{E}-09$ & $-1.83169454607581 \mathrm{E}-12$ & $1.85525846448335 \mathrm{E}-12$ \\
\hline Algorithmic & $-1.91045438660579 \mathrm{E}-09$ & $-1.83169454607586 \mathrm{E}-12$ & $1.85525846448341 \mathrm{E}-12$ \\
\hline Relative Error & $-1.58 \mathrm{E}-14$ & $-2.73 \mathrm{E}-14$ & $3.24 \mathrm{E}-14$ \\
\hline
\end{tabular}




\section{Conclusions and Future Work}

This paper reports on significant advancements of the aeroacoustic analyses and sensitivities in the open-source software SU2. The previous aeroacoustic analysis, suitable for fixed noise sources and a wind tunnel Ffowcs Williams and Hawkings formulation, has now been extended for the full Farassat's formulation F1A. This extension enables predictions of far-field noise generated by moving sources.

The new formulation is verified for a stationary and rotating sphere in a wind tunnel and for a tiltrotor in forward flight, by comparing SU2 solutions with the predictions computed by NASA's aeroacoustics code ANOPP2. The algorithmic capability of SU2 provides discretely consistent, adjoint-based sensitivity analysis for the F1A formulation. The adjoint-based sensitivities are compared with the complex-step sensitivities and found practically identical as expected.

The future work will focus on the extension of the SU2 adjoint framework to sliding mesh multizonal computations. This capability will enable the analysis and sensitivity of the aeroacoustics associated with the propeller, which is in relative motion with respect to stationary surfaces. A moving mesh associated with the propeller will be in relative motion with respect to a stationary mesh. Another future direction is to use the SU2 multidisciplinary framework for efficient design of mounted propellers. This work will include step-by-step optimization of the propeller placement delivering the required thrust with significant reduction of generated noise.

\section{Acknowledgment}

This work was supported by a NASA Langley Research Center grant through the National Institute of Aerospace.

\section{References}

1. Jameson, A. "Aerodynamic design via control theory," Journal of Scientific Computing Vol. 3, No. 3, 1988, pp. 233-260.

doi: $10.1007 / \mathrm{bf} 01061285$

2. Pironneau, O. "On optimum design in fluid mechanics," Journal of Fluid Mechanics Vol. 64, No. 1, 1974, pp. $97-110$. doi: $10.1017 / \mathrm{S} 0022112074002023$

3. Baysal, O., and Ghayour, K. "Continuous Adjoint Sensitivities for Optimization with General Cost Functionals on Unstructured Meshes," AIAA Journal Vol. 39, No. 1, 2001, pp. 48-55. doi: 10.2514/2.1269

4. Nielsen, E. J., Lee-Rausch, E. M., and Jones, W. T. "Adjoint-Based Design of Rotors in a Noninertial Reference Frame," Journal of Aircraft Vol. 47, No. 2, 2010, pp. 638-646. doi: $10.2514 / 1.46044$

5. Nielsen, E. J., and Diskin, B. "Discrete Adjoint-Based Design for Unsteady Turbulent Flows on Dynamic Overset Unstructured Grids," AIAA Journal Vol. 51, No. 6, 2013, pp. 1355-1373.

doi: 10.2514/1.J051859

6. Rumpfkeil, M., and Zingg, D. "Unsteady Optimization Using a Discrete Adjoint Approach Applied to Aeroacoustic Shape Design," 46th AIAA Aerospace Sciences Meeting and Exhibit. American Institute of Aeronautics and Astronautics, 2008.

7. Fabiano, E., and Mavriplis, D. "Adjoint-Based Aeroacoustic Design-Optimization of Flexible Rotors in Forward Flight," Journal Of The American Helicopter Society Vol. 62, No. 4, 2017. doi: 10.4050/JAHS.62.042005

8. Economon, T., Palacios, F., and Alonso, J. "A Coupled-Adjoint Method for Aerodynamic and Aeroacoustic Optimization," 12th AIAA Aviation Technology, Integration, and Operations (ATIO) Conference and 14th AIAA/ISSMO Multidisciplinary Analysis and Optimization Conference. 2012.

9. Economon, T. D., Palacios, F., and Alonso, J. J. "An Unsteady Continuous Adjoint Approach for Aerodynamic Design on Dynamic Meshes," 15th AIAA/ISSMO Multidisciplinary Analysis and Optimization Conference. 2014.

10. Baysal, O., and Eleshaky, M. E. "Aerodynamic design optimization using sensitivity analysis and computational fluid dynamics," AIAA Journal Vol. 30, No. 3, 1992, pp. 718-725.

doi: $10.2514 / 3.10977$ 
11. Eleshaky, M. E., and Baysal, O. "Shape Optimizing Nacelle near Flat-Plate Wing Using Multiblock Sensitivity Analysis," Journal of Aircraft Vol. 35, No. 1, 1998, pp. 33-38.

doi: $10.2514 / 2.2286$

12. Economon, T. D., Palacios, F., Copeland, S. R., Lukaczyk, T. W., and Alonso, J. J. "SU2: An Open-Source Suite for Multiphysics Simulation and Design," Vol. 54, No. 3, 2016, pp. 828-846.

doi: 10.2514/1.J053813

13. Zhou, B., Albring, T. A., Gauger, N. R., Ilario, C., Economon, T. D., and Alonso, J. J. "Reduction of Airframe Noise Components Using a Discrete Adjoint Approach," 18th AIAA/ISSMO Multidisciplinary Analysis and Optimization Conference. 2017.

14. Zhou, B. Y., Albring, T. A., Gauger, N. R., Economon, T. D., and Alonso, J. J. "An Efficient Unsteady Aerodynamic and Aeroacoustic Design Framework Using Discrete Adjoint," 17th AIAA/ISSMO Multidisciplinary Analysis and Optimization Conference. 2016.

15. Zhou, B. Y., Albring, T. A., Gauger, N. R., Economon, T. D., Palacios, F., and Alonso, J. J. "A Discrete Adjoint Framework for Unsteady Aerodynamic and Aeroacoustic Optimization," 16th AIAA/ISSMO Multidisciplinary Analysis and Optimization Conference. 2015.

16. Farassat, F. "Derivation of Formulations 1 and 1A of Farassat," NASA/TM-2007-214853. NASA Langley Research Center, 2007.

17. Lopes, L., and Burley, C. "Design of the Next Generation Aircraft Noise Prediction Program: ANOPP2," 17th AIAA/CEAS Aeroacoustics Conference (32nd AIAA Aeroacoustics Conference). 2012.

18. Lopes, L., and Burley, C. "ANOPP2 User's Manual: Version 1.2," NASA/TM-2016-219342. NASA Langley Research Center, Hampton, Virginia, 2016.

19. Jameson, A. "Origins and Further Development of the Jameson-Schmidt-Turkel Scheme," AIAA Journal Vol. 55, No. 5, 2017, pp. 1487-1510.

doi: 10.2514/1.J055493

20. Sagebaum, M., Albring, T., and Gauger, N. "High-Performance Derivative Computations using CoDiPack," arXiv.org, 2017.

21. Williams, J. E. F., Hawkings, D. L., and Lighthill, M. J. "Sound generation by turbulence and surfaces in arbitrary motion," Vol. 264, No. 1151, 1969, pp. 321-342.

doi:10.1098/rsta.1969.0031

22. Francescantonio, P. D. "A new boundary integral formulation for the prediction of sound radiation," Journal of Sound and Vibration Vol. 202, No. 4, 1997, pp. 491-509.

23. Achenbach, E. "Experiments on the flow past spheres at very high Reynolds numbers," Journal of Fluid Mechanics Vol. 54, No. 3, 1972, pp. 565-575.

doi: $10.1017 / \mathrm{S} 0022112072000874$

24. "Advancement of proprotor technology. Task 1: Design study summary," NASA-CR-114682. Bell Helicopter Co., 1969.

25. "Advancement of proprotor technology. Task 2: Wind-tunnel test results," NASA-CR-114363. Bell Helicopter Co., 1971.

26. Marr, R. L. "Wind Tunnel Test Results of 25 Foot Tilt Rotor During Autorotation," NASA-CR-137824. Bell Helicopter Co., 1976.

27. Hanson, D. "The influence of propeller design parameters on far field harmonic noise in forward flight," 5th Aeroacoustics Conference. 2012.

28. Hubbard, H. H. Aeroacoustics of Flight Vehicles: Theory and Practice. Volume 1: Noise Sources. Langley Research Center: NASA, 1991.

29. Squire, W., and Trapp, G. "Using Complex Variables to Estimate Derivatives of Real Functions," SIAM Review Vol. 40, No. 1, 1998, pp. 110-112.

doi: $10.1137 / \mathrm{s} 003614459631241 \mathrm{x}$

30. Martins, J. R. R. A., Sturdza, P., and Alonso, J. J. "The complex-step derivative approximation," ACM Trans. Math. Softw. Vol. 29, No. 3, 2003, pp. 245-262.

doi: $10.1145 / 838250.838251$ 